\title{
CREAR UN ESPACIO DE LECTURA
}

\author{
Adriana Cabrera Tejada \\ Maestría En Educación \\ Universidad Sur colombiana
}

La Asociación Colombiana de Universidades, comprometida con el mejoramiento de la calidad de la educación superior, considera el tema de la lectoescritura como uno de los más urgentes y relevantes. En efecto en educación superior, la lectura y la escritura no se pueden separar de asuntos tan sensibles como la del abandono, el rendimiento académico, el fortalecimiento de la investigación, la producción de conocimiento y el desarrollo de competencias laborales, entre otros, que representan, hoy por hoy, criterios básicos para los procesos de acreditación.

Las investigaciones recientes en este campo han mostrado que los diversos saberes requieren de diferentes prácticas lectoras y escriturales, las cuales, a su vez, precisan de procesos formativos específicos. Así, se viene reconociendo cada vez más que tales procesos no son exitosos cuando, como ocurre tradicionalmente, sólo se los deja a cargo de los profesores de lengua y comunicación. Las dificultades que la universidad enfrenta en este terreno, no puede ser resultados sin una política que comprometa a todas las personas implicadas en el formación universitaria.

Cuando se estudian las estadísticas de lectura lo que se observa realmente es la complejidad de un país. Una exploración de los datos cuantitativos de los módulos de hábitos de lectura de la encuesta continua de hogares (2002 y 2005) confirma dos grandes realidades de Colombia: su amplia diversidad y su enorme desigualdad.

Por una parte está la diversidad, por ejemplo, la lectura de los jóvenes frente a la lectura de los adultos, la de las mujeres frente a la de los hombres. La de los jóvenes, que leen más de lo que habitualmente se supone, es una lectura marcada fundamentalmente por el deber y las exigencias escolares, abierta a las nuevas tecnologías y muy vinculada con la música y la televisión. Los adultos, a diferencia de los jóvenes, se interesan por la lectura de periódicos, los libros de auto superación y, a medida que aumenta la edad, los libros religiosos.

La lectura también es un asunto de género. Las mujeres leen más que los hombres y tienen a su favor una cualidad extraordinaria: los niños estiman que sus madres son las personas más adecuadas para leerles en voz alta y las que ocu- 
pan el primer lugar como promotoras del hábito de leer. La mujer, además de lectora es fundamental en la reproducción social de la lectura.

Pero la diversidad de las lecturas es aún mayor y más rica. Se leen libros y revistas, periódicos y textos escolares. Pero también manuales, historietas, cómics y folletos. Se lee sobre soporte físico, pero cada vez más sobre soporte digital. $Y$ al hacerlo se entremezclan, de una manera creativa, el texto escrito con el video, la imagen fotográfica con las infografías y los sonidos.

Uno de los datos que más asombró a los colombianos fue el desarrollo de la lectura en Internet, la única que creció en Colombia. En sólo cinco años las cifras se doblaron y en las 13 principales ciudades del país se pasó del 5\% al $11 \%$. En Bogotá los resultados fueron aún más abrumadores: prácticamente se triplicaron. Pero lo más interesante del aumento es que esto sucedía mientras que el promedio de lectura de libros en Colombia se desplomaba el 33\%, pasando de 2.4 libros leídos al año a 1.6.

De ahí que la capacitación de los docentes debe ser atendida sin demora y la actividad tutorial se ve implicada, pues ésta genera un contexto muy propicio para detectar los problemas y encontrar alternativas de solución a estos desafíos educativos. Se acepta ampliamente que la capacidad de leer, y de aprender de la lectura, es una habilidad académica fundamental de gran importancia para él éxito escolar en cualquier área de estudio y en todos los niveles educativos. Específicamente, parece claro que los estudiantes con deficiencias en la lectura tienden a presentar correlativamente deficiencias académicas múltiples, a diferencia de estudiantes con otro tipo de deficiencias básicas.

Por ello, el desarrollo de las habilidades de lectura y escritura en los estudiantes universitarios requiere de concepciones y propuestas pedagógicas realmente eficaces y eficientes. Dentro de ésta, el estudiante como usuario novicio de modalidades académicas de la lengua escrita requiere de un apoyo y una retroalimentación decididas de los tutores como conocedores de la comunicación escrita en su disciplina, capaces de apoyar el desarrollo de las habilidades que corresponden a su manejo.

Así mismo este es un tema que no ha sido tọcado en su magnitud en las universidades, propende por resaltar su importancia para iniciar con un proceso necesario de investigación, capacitación, actualización y gestión, en un intento por aportar elementos para responder a esta necesidad, se realizó una tarea de desarrollar ambientes de capacitación que interpelen a los docentes en tres direcciones relacionadas: primero como individuos lectores y productores de textos escritos; segundo, como profesores, investigadores y difusores del co- 
nocimiento disciplinario e interdisciplinario, conocimiento cuyo formato básico es el escrito; y tercero, como tutores que tienen la responsabilidad de acompañar y apoyar a sus tutorados hacia la exitosa culminación de sus estudios profesionales, con base en un sólido manejo de la lengua escrita no sólo como un instrumento de comunicación, sino también de expresión y pensamiento.

En Colombia la educación ha estado inmersa en un proceso de evolución, en el cual se han dado cambios considerables para su mejoramiento, han surgido herramientas para la enseñanza, tratando de lograr el desarrollo de un aprendizaje significativo que contribuya con la transformación que el país necesita.

Aunque en Colombia se cuenta con políticas públicas educativas, que pretenden mejorar la calidad de la educación, los procesos educativos siguen estancados en un momento histórico que no está acorde con las necesidades e intereses que tienen las acciones de mejoramiento; apoyados en el análisis de los referentes teóricos existentes y la convicción de los educadores hacia el cambio en el quehacer educativo.

En la actualidad, dentro del mundo académico es importante en toda sociedad civilizada que el profesional debe estar preparado para el cambio constante de nuestro mundo, el profesional debe ser competitivo, por otro lado debe conocer sus estudiantes y su comunidad, siendo ellos los que esperan que desde la educación se empiecen a forjar a ciudadanos competentes y formados en la búsqueda del mejoramiento de la calidad de vida de cada uno de ellos y de la sociedad.

¿Por qué se nos dificulta tanto la lectura? ¿Es la lectura tan aburrida como imaginamos? Estas son algunas de las muchas preguntas que nos formulamos constantemente, cuando tal vez la pregunta correcta a formularnos sea ¿Realmente sé como leer? Y posiblemente la respuesta a esta pregunta sea: no.

La comprensión es La capacidad que posee cada uno de entender y elaborar el significado de las ideas relevantes de textos escritos de distinta naturaleza, asimilando, analizando e interpretando el mensaje que el texto contiene y relacionarlas con las ideas que ya se tienen. Esta capacidad hace parte del proceso de descodificación de un texto. Los estudiantes encuentran un gran problema a la hora de descodificar, porque hay subdesarrollo de los procesos mentales cuando se lee.

En el proceso de lectura se utilizan un sin número de estrategias para la comprensión de lo que se lee y algunos estudiantes las utilizan y aprovechan al máximo, en cambio hay estudiantes que desconocen tales estrategias y tampoco saben cómo aprenderlas, es aquí donde interviene la meta cognición 
que se entiende como el control que tiene el sujeto de sus destrezas o procesos cognitivos, de pensamiento y de la habilidad para dar y darse cuenta de estos procesos a la hora de leer; pero el problema con estos estudiantes es realmente el Subdesarrollo y desconocimiento de las habilidades meta cognitívas. Sin lugar a duda, se ha reconocido que muchos de los problemas de comprensión y de aprendizaje que presentan los estudiantes se deben a la falta de eficiencia en el empleo de la Meta cognición. En consecuencia, carecen de la habilidad para utilizar estrategias efectivas al enfrentar textos, no vigilan y regulan el aprendizaje, no se forman una imagen mental acerca de que va a leer, como lo va a hacer, si tiene algún conocimiento previo acerca del tema y para qué lo hará. Algunos estudiantes, desarrollan por si mismos estas habilidades de una forma eficiente, pero desafortunadamente otros no, así que el objetivo principal del presente trabajo será plantear habilidades meta cognitivas y estrategias de lectura para mejorar y desarrollar la comprensión de lectura. Para lograr este objetivo primero se describirá el proceso de lectura y se explicaran algunas de las estrategias de lectura que hacen posible la comprensión de un texto; después se planteará la comprensión de lectura como finalidad de todo el proceso de lectura y por último abarcando los pasos anteriores se describirán cada una del las habilidades meta cognitivas para darle solución al problema de subdesarrollo de estas habilidades en los lectores.

Igualmente uno de los objetivos principales de la educación básica y media es que el estudiante pueda usar la lectura y la escritura como herramientas básicas para aprender, es decir que sea capaz de acceder a la información de manera eficiente, de evaluarla crítica y competentemente, y de usarla correctamente para producir sus propios conocimientos.

Los resultados de las pruebas de estado y de las pruebas Saber demuestran que a pesar de los esfuerzos que se han hecho, el trabajo de lectura y escritura que se lleva a cabo en las aulas del país, no es aún el más adecuado. Al terminar su formación básica y media, los estudiantes saben leer pero, con frecuencia, no comprenden, ni manejan, ni asimilan lo que leen.

Por consiguiente para lograr lectores autónomos y críticos es importante que los estudiantes aprendan a manejar los textos de una manera adecuada que les incentive el pensamiento deductivo, les permitan establecer relaciones y los motive al trabajo interdisciplinario.

Igualmente convertir la lectura y la escritura en las actividades básicas de la construcción del conocimiento, requiere que desde el preescolar se forjen las bases para que los niños se acerquen a ellas con agrado y desarrollen no sólo una buena mecánica de los procesos, sino que fortalezcan la comprensión y 
el análisis, utilicen la escritura como una herramienta fundamental de comunicación y hagan de la discusión una práctica permanente para confrontar y enriquecer sus ideas.

Teniendo en cuenta lo anterior todas las instituciones deberían dar pie a la implementación de un Proyecto de Lectura buscando:

1. Darle a los maestros las bases teóricas y las herramientas concretas para que puedan utilizar fácil, lúdica y creativamente los libros de la colección de la biblioteca y la sala de lectura, para el desarrollo de las cuatro habilidades comunicativas: (leer, escribir, hablar y escuchar) y los tres niveles de lectura (literal, inferencial y crítico), en los estudiantes.

2. Motivar a los estudiantes a que lean, informándolos acerca de los libros que pueden encontrar en la biblioteca y el salón de la lectura según sus intereses y su edad, con un material lúdico y divertido.

Por consiguiente la experiencia acumulada por quienes tienen a cargo el diseño técnico del Proyecto y sobre todo del trabajo directo con maestros y los alumnos de diferentes grados de la institución, permitirá perfilar el enfoque y la fundamentación metodológica del mismo, a partir de realidades concretas y teniendo como base las principales deficiencias encontradas.

El desconocimiento por parte de los maestros de cómo se trabajan los textos para desarrollar de manera creativa y eficiente la comprensión lectora de los estudiantes. En muchas ocasiones es común encontrar maestros que no leen; que no entienden bien los estándares de lenguaje del Ministerio o no saben cómo hacer para alcanzarlos; que no conocen muchas obras de literatura infantil y juvenil y por lo tanto no saben qué lecturas sugerirle a sus estudiantes, o no se les ocurren otras actividades alrededor del libro diferentes a que elaboren un resumen del argumento y de la biografía del autor.

Así La falta de un conocimiento práctico por parte de los usuarios y docentes en el uso, estructura interna, espacio, colección, forma de acceder a los libros, la falta de material interesante, de buena calidad y adecuado a las diferentes edades, a partir del cual se pueda desarrollar la comprensión lectora y la no preparación del maestro para utilizar ciertos métodos alternativos de aprendizaje como la lectura en voz alta, la creatividad manual, la experimentación o el trabajo en equipo; hacen de estas limitaciones se consideren como el enfoque del proyecto debía apuntar a convencer a los maestros de que el trabajo en las bibliotecas puede y debe ser no solo una actividad lúdica y gratificante sino también una experiencia articulada a sus programas de clase y dirigida a alcanzar los estándares de calidad definidos por el Ministerio de Educación. 
A partir de estos supuestos se definen algunas características de la metodología para el desarrollo de un proyecto así:

1. Buscar ante todo la gratificación espiritual y lúdica, desde la lectura con una metodología basada en el juego y el divertimento.

2. Las guías de trabajo se elaborarían de manera llamativa y divertida, con propuestas para acercarse a la lectura desde la oralidad, el juego, el arte, las manualidades, el baile, la música y las muchas formas de representación creativa.

3. Trabajar la lectura y las visitas a la sala de lectura como parte de los programas de clase, de manera que no exige tiempo extra por parte del maestro.

4. El proyecto pretende motivar al maestro a que él también lea, conozca, promueva y visite frecuentemente la biblioteca y la sala de lectura. La metodología apela directamente a su experiencia como lector para que trabaje con sus alumnos desde ella.

5. El material entregado al maestro durante la capacitación debe darle seguridad frente al bibliotecario y frente a sus alumnos sobre el manejo de la biblioteca y de la colección y por supuesto la visita a la sala adecuada para la lectura.

6. El trabajo y las actividades propuestas en los guías buscan crear condiciones para ayudarle al maestro a alcanzar los estándares de calidad definidos por el Ministerio de Educación.

7. El método de trabajo propuesto busca ayudarle al maestro a conocer los gustos e intereses de sus alumnos para lograr un aprendizaje șignificativo El proyecto busca convertirse en catalizador y facilitador de las relaciones entre estudiantes y maestros y de los estudiantes entre sí.

8. Los talleres de capacitación preparan al maestro para utilizar ciertos métodos alternativos de aprendizaje como la lectura en voz alta, la creatividad manual, la experimentación o el trabajo en equipo.

Por consiguiente como apertura de este programa se realiza un evento de presentación del programa, con la participación de las autoridades locales, los directivos docentes, los amigos de la lectura y los líderes del área cultural, esto con el propósito de dar a conocer formalmente el proyecto a la comunidad.

En conclusión el proceso de lectura va desarrollando habilidades para leer desde pequeños, pero a veces estas habilidades no se desarrollan porque no 
se educa en lectura a los jóvenes; por tal motivo cuando crecen se encuentran con un gran problema "no se puede comprender un texto" por eso he planteado a la meta cognición como solución a este problema ya que los estudiantes no tienen conocimiento de las estrategias lectoras que pueden utilizar. La mejor forma de desarrollar estas habilidades es practicando cada una de las estrategias propuestas y enfatizar en la repetición del proceso de lectura tantas veces como se pueda; solo así se puede llegar a un conocimiento de sus propios procesos mentales.

Incluso la comprensión de lectura es el objetivo final de la lectura y el objetivo inicial es la expresión escrita ya que para leer algo, antes ya debió estar escrito por un autor, que quería dar a conocer sus puntos de vista y comunicarlos a través de este medio. En la comprensión existen factores relacionados con el lector y con el texto que dificultan la creación de un significado propio de lo que quiere decir el autor si el lector no tiene las habilidades de comprensión suficientes para hacer inferencias y obtener un aprendizaje de lo que lee.

Es evidente que las dificultades en relación con la lectura y la escritura son el factor determinante en el tema del abandono o el bajo rendimiento académico. Pero sí es claro que la urgencia de una política de alfabetización en cada institución educativa supera el hecho de que el ámbito universitario exija el dominio de nuevas siluetas textuales y se relaciona con aspectos tan sensibles para la vida universitaria como el fortalecimiento de la investigación, la producción de conocimiento y con un problema mucho más sofisticado y fundamental: la exclusión. Miles de estudiantes pueden estar impedidos para ingresar a una universidad o avanzar en el currículo que esta propone por dificultades emparentadas con el proceso de leer y escribir.

Es evidente que la debilidad de los procesos que tuvieron los hoy estudiantes universitarios, reflejada, en su formación primaria y secundaria y su entorno familiar, fijando los bajos niveles de comprensión y sustentación de lo leído. Se encuentra mucha dificultad con la técnica lectora, por que las bases que traen los estudiantes y la falta de hábitos de estudio, impiden que se generen procesos en la universidad.

Es determinante que el nivel de lectura en el proceso universitario no ha tenido los resultados esperados, faltan estrategias pedagógicas para la adopción de parámetros especíicos del buen uso de la lectura y escritura; De otra parte, los que aprenden a leer, si no practican habitualmente la lectura terminan olvidando mucho de lo aprendido y, no cumplen las condiciones que exige el mundo globalizado y cada vez son más dependientes de las altas tecnologías, ello comprueba los bajos niveles de lectura y escritura de los estudiantes universitarios. 


\section{BIBLIOGRAFÍA}

ARGUELLES, Antonio. "Competencia laboral y educación basada en normas de competencia", México, Limusa 1996.

BOJACA, B, VADORI, G, NOVOA, P (2001)" Los procesos de lectura y escritura como estrategias de formación docente", En: La formación en América Latina. Bogotá: Editorial Magisterio.

BRUNER, J. (1989). Acción, pensamiento y lenguaje. Madrid: Alianza Editorial.

CARLINO, P. y Estienne, V. (2004). ¿Pueden los universitarios leer solos? Un estudio exploratorio. Trabajo presentado en las XI Jornadas de Investigación en Psicología, Buenos Aires. (2004b). Leer en la universidad: enseñar y aprender una cultura nueva. Extraído el 11 de febrero de 2008 desde http://unipluriversidad. udea.edu.co/vol4-1/vol4-3-2.htm Carlino, P. y Diment, E. (2006). Perspectivas de alumnos y docentes sobre la escritura en los primeros años de la universidad. Un estudio piloto. Trabajo presentado en las XIII Jornadas de Investigación en Psicología, Buenos Aires.

CARRIEDO, N. Y Alonso, J. (1991): Enseñanza de las ideas principales: problemas en el... Cassany, D. (1987): Describir el escribir. Cómo se aprende a escribir....

CARVAJAL, G. (2008). Lecturas y escrituras juveniles. Entre el placer, el conformismo y la desobediencia. Colección Libros de Investigación. Cali: Universidad del Valle.

CINTERFOR-OIT "formación y Trabajo: de ayer para mañana", papeles Técnicos No. 1, Montevideo, Uruguay, 1996.

CINTERFON-OIT “Horizontes de la formación. Una carta de navegación para los países de América Latina y el Caribe" XXXII reunión de la Comisión Técnica, Ocho Ríos, Jamaica, 1995.

COLOMBIA. MINISTERIO DE EDUCACIÓN NACIONAL. Tendencias Mundiales AI Tablero. No 37, oct-dic. Santa Fe de Bogotá. 2005. p. 9

COLOMBIA, SECRETARIA DE EDUCACION DISTRICTAL (2009). Evaluando ciclo a ciclo. Lineamientos generales. Disponible en: http://redacademica.redp.edu. co/evaluación/attachments/015_ciclo_ciclo_ok.pdf́.Recuperado el 06 de julio de 2010.Bogotá.

COLOMER, T. y Camps, A. (1996): Enseñar a leer, enseñar a comprender. Celeste/ MEC. Madrid. 
FERREIRO, E. (2001).Pasado y presente de los verbos leer y escribir. México: Fondo de Cultura Económica.

FERREIRO, E. y Teberosky. A. (1979): Los sistemas de escritura en el desarrollo del niño. Siglo XXI. México.

FERREIRO, E. (2002). "Leer y escribir en un mundo cambiante". Disponible en: http//www.craaltaribagorza.net/article.php3id_article=895.Recuperado el 10 de abril de 2010.

GARCIA F. (2006). Las tecnologías de la información y la comunicación y la pedagogía de medios. Hacia la construcción de una nueva competencia mediática. Berlín: Universidad de Berlín.

GONZÁLEZ, B. (2006). Experiencia de Alfabetización Académica en la Universidad Sergio Arboleda de Colombia. Trabajo presentado en el Primer Congreso Nacional "Leer, escribir y hablar hoy", septiembre, Tandil. (2008a). Dispositivos para pensar una política institucional para el desarrollo de la lectura y la escritura en la educación superior. Conferencia presentada en la Universidad Autónoma Metropolitana de México, México D.F.

GOODMAN, K, (1996) “¿Cómo la enseñanza apoya el aprendizaje en el desarrollo de la lectoescritura"? En: Rodríguez, M.E (1996).Alfabetización para todos y para todos. Buenos Aires: Aique.

GOODMAN, K. (1996). La lectura, la escritura y los textos escritos: una perspectiva transaccional socio psicolingüística. Textos en Contexto, 2, 21-27.

JURADO, F; BUSTAMANTE, G.y PEREZ, M. (1988).Juguemos a interpretar, evaluación de competencias en la lectura y escritura. Bogotá: Plaza y Janés.

LANGER, J. (1992)"La lectura, la escritura y el desarrollo de géneros". En: Irwin, J.Y Doyle, M.A. (campus.) Conexiones entre la lectura y la escritura. Buenos Aires: Aique.

LERNER, D (2001). Leer y escribir en la escuela: lo real, lo posible y lo necesario. México: Fondo de Cultura Económica.

LOMAS, Carlos y Amparo Tusón (2009): Enseñanza del lenguaje, Enseñanza del lenguaje, emancipación comunicativa y educación crítica. Editorial: Manual Moderno S.A. de C. V. (México, D.F.)Eděre S.A. de C. V. Año de edición: 2009. 


\section{CONCLUSIONES DE LA INVESTIGACIÓN}

Las siguientes conclusiones comenzarán intentando responder a las preguntas iníciales que guiaron esta investigación, para luego dar una mirada global a través de conclusiones de carácter más amplio.

¿Cuáles es la aptitud y actitud de los docentes en el proceso de enseñanza aprendizaje de inglés con los estudiantes?

La mayor parte de los docentes del área, se destaca por tener un buen nivel dentro del estándar usuario independiente B1; sin embargo no es el más alto para realizar los procesos de enseñanza - aprendizaje con los estudiantes de manera eficaz; especialmente en lo referente a las habilidades: comprensión lectora, verbales y de escucha.

Se observaron limitaciones y tensiones en la competencia didáctica y metodológica de los docentes en el momento de clase; puede ser porque el área de inglés se compone mayormente de docentes con especialidad diferente a los licenciados en inglés que no han recibido la suficiente formación pedagógica especializada para la enseñanza de otro idioma.

¿Cuál es la aptitud de los estudiantes hacia el manejo del idioma inglés en este contexto en particular?

Los estudiantes del grado noveno han trabajado los procesos de inglés por más de 300 horas estudio, y de acuerdo con los estándares nacionales se necesita un promedio de 250 horas para que una estudiante se desempeñen acorde al nivel $A 2$ del MCE, sin embargo, los resultados obtenidos en los estudiantes de la I.E. fueron muy bajos, demostrando así, que el nivel de dominio que tiene los estudiantes es bajo en referencia al grado escolar, edad y tiempo de trabajo en el proceso.

De la misma forma los estudiantes de grado $11^{\circ}$ vistos como las personas en quienes se puede observar el éxito de los procesos de las diferentes áreas de estudio; en lo referente al inglés, presentan un nivel muy bajo a pesar de haber estudiado en secundaria y media (aproximadamente 700 horas); y en este grado los estudiantes no tienen los fundamentos necesarios para desempañarse en el nivel B1 de MCE, nivel para el cual se necesita entre 350 a 450 horas de estudio, en nuestro país.

Otro referente del nivel de inglés son los resultados de las pruebas Saber 11 de los años anteriores; donde se puede analizar que hay un porcentaje promedio de $52 \%$ de los estudiantes que presentaron la prueba de inglés, obtuvieron un 
nivel de A- de acuerdo con los rangos del lcfes, rango que está por debajo del nivel Básico A1 y que no lo estipula ningún estándar nacional o internacional.

¿Cómo participan las directivas de las instituciones educativas en el desarrollo de los planes y proyectos que promueve el Estado, hacia el dominio del idioma inglés?

El rol de los directivos docentes de las I.E. ha cambiado significativamente, y en la I.E objeto de estudio no fue la excepción. Las directivas realizan todos sus esfuerzos por mantener lo que se tiene y lo poco que se pueda tener en la institución: planta física, cobertura, convenios, servicios y cuadros estadísticos que realizan diariamente; los cuales son gestados y controlados por el Ministerio de Educación Nacional o por las Secretarías de Educación Departamental o Municipal.

Por otro lado, los docentes directivos no se involucran ni participan plenamente en los procesos académicos, de evaluación ni de formación que realiza el área de inglés, siendo entonces docentes del área los responsables de todas las acciones pedagógicas que se realicen en la I.E. Igualmente las respuestas dadas en la encuesta por los directivos no coinciden con las opiniones que dan los docentes; ni tampoco con los resultados de los estudiantes evaluados en la institución educativa.

¿Cuál es el impacto de los planes y programas en el mejoramiento del nivel del idioma inglés?

Desde el año 2006 esta I.E. ha recibido apoyo gubernamental, en cuanto a soporte económico y también ha contado con asesoría especializada y capacitación en pro de consolidar y mejorar el proceso de enseñanza aprendizaje del idioma inglés acorde con los estándares municipales, nacionales e internacionales, pero se observa que el nivel de inglés de los estudiantes ha mejorado muy poco, demostrando así un impacto bajo.

A pesar del trabajo permanente de los docentes del área de inglés, las metas estipuladas por el MEN, sobre el nivel de dominio que deberían tener los docentes y estudiantes a 2019, no se van a lograr en las condiciones de las instituciones educativas y de los procesos que se están llevando a cabo en las aulas escolares.

¿Cuáles son las prácticas pedagógicas más utilizadas actualmente por los docentes de la institución educativa, en el proceso de enseñanza - aprendizaje del inglés? 
Vale la pena tener en cuenta el concepto de práctica pedagógica entendida según Valencia (2000) como "la actividad profesional del docente derivada de métodos y procedimientos con un fondo investigativo y de conocimientos teóricos elaborados sistemáticamente", pues dicha definición nos permite ubicarnos alrededor de las actividades que llevaron a cabo con sus estudiantes en el contexto escolar.

Así entonces, podemos afirmar que las prácticas pedagógicas más utilizadas por los docentes de Inglés son las actividades Pre-comunicativas y actividades Comunicativas (Littlewood, 1998).

Actividades Pre-comunicativas: el docente se centró en elementos específicos del conocimiento o habilidades y trató de brindar oportunidades para que los estudiantes las practicaran separadamente. Dichas actividades de tipo estructural y cuasi-comunicativo, tales como drills y hacer y responder preguntas, las cuales podemos encontrar en la mayoría de libros de texto.

En relación con las actividades comunicativas, estas procuraban que el estudiante integrara sus conocimientos y habilidades pre-comunicativas para poder usarlas en la comunicación de significados, de manera que el estudiante se comprometiera aun más en la práctica de la comunicación. No hay conexión entre lo que se proyecta alcanzar y la realidad de los estudiantes, pues pareciera que el inglés se asumiera como una asignatura más.

La mayoría de profesores tienen competencia comunicativa en inglés, pero demuestran limitaciones al enseñarlo, dado que no hay participación activa del estudiante y por el contrario el docente se vuelve el protagonista.

La enseñanza aprendizaje del inglés debe enfocarse hacia los principios y enfoques comunicativos y metodológicos, en vez de la acción de clase en la gramática.

No se tienen diseñados los espacio lúdicos, tecnológicos y físicos donde haya la necesidad de mantener una comunicación continua; como apoyo para el aprendizaje del idioma; como: Música, cine, libros, periódico, clubes e inmersiones.

El proceso de inglés inicia en los niños y niñas desde el grado $6^{\circ}$, entorpeciendo todo el proceso de bilingüismo que pretende alcanzar las políticas públicas del estado. Así, no se aprovecha el interés de los jóvenes. 
El aprendizaje de la lengua se realiza en un contexto aislado al desarrollo del contexto cultural del mismo necesario para que los estudiantes vean de manera significativa el aprendizaje. Su énfasis debe radicar en el significado antes que en la forma y la corrección

Finalmente, se pretende abrir una brecha para que estudios posteriores busquen alternativas de intervención que se puedan abordar desde las mismas aulas y verificar su efíciencia en cuanto al mejoramiento de estrategias y prácticas pedagógicas y orientaciones curriculares a la luz del diagnóstico obtenido que propendan por una mayor eficiencia en los procesos de enseñanza - aprendizaje del inglés. Dichas alternativas deben ejecutarse y evaluar sus resultados para unificar criterios en cuanto a actividades de clase y tipos de competencias que éstas promueven, con el fin de influir en el medio educativo local y regional para cualificar y enriquecer la labor de los docentes de inglés y por ende del aprendizaje en los alumnos.

De hecho las políticas públicas alrededor de los procesos de inglés son oportunidades para que todas las instituciones educativas en los diferentes niveles hagan uso de las pedagogías y/o teorías; que generen mejoría del proceso de adquisición de un segundo idioma, en un contexto real. El hecho de tener como base el Marco Europeo, con todas sus exigencias de nivel internacional, no quiere decir que se tiene que perder nuestra identidad y cultura y que la actitud hacia el bilingüismo sea menos positiva que antes. Sin embargo, se hace primordial la investigación y la auto-evaluación continua del Programa para que se pueda dar una retroalimentación pertinente a cada proceso y de esa forma ver resultados y transformaciones reales en los participantes.

\section{BIBLIOGRAFÍA}

ABBGNANO y Visalberghi. En: Los fundamentos desde la pedagogía, la metacognición, y las competencias. 1995. p. 61

ASHER, James J. The Total Physical Response Approach to Second Language Learning. The Modern Language Journal, Vol. 53, No. 1. 1969.

AUSUBEL, David. The Psychology of Meaningful Verbal Learning. Grune \& Stratton. New York. 1963.

BAILEY, K. M. (2006). Language Teacher Supervision. Cambridge University Press.

BAKER, C. Key Issues in Bilingualism and Bilingual Education. Clevedon: Multilingual Matters. 1988. P112. 
BRITISH COUNCIL, English exams: starters, movers, flyers, key and pet - Cambridge University Press.

BROWN, D. (2000). Principles of Language Learning and Teaching. $4^{\text {th }}$ Ed. Pearson Longman.

CARPIO BEGAZO, Álvaro. Pautas para aprender mejor. http://alvaro.carpiobegazo.googlepages.com /

COLOMBIA. MINISTERIO DE EDUCACIÓN NACIONAL (MEN) Colombia Bilingüe. En: Periódico AL TABLERO. No 37, octubre-diciembre. Bogotá.2005.

COLOMBIA. MINISTERIO DE EDUCACION NACIONAL. Estándares Básicos de Competencias en Lenguas Extranjera. Op. Cit. p.2.

COLOMBIA. MINISTERIO DE EDUCACIÓN NACIONAL. Lineamientos Curriculares. Idiomas extranjeros. Santa Fe de Bogotá. 1999. p. 132

COMENIO, Juan Amós. En: Los fundamentos desde la pedagogía, la metacognición, y las competencias. 1657. p.64

CONSEJO DE EUROPA. Marco de Referencia Europeo: Aprendizaje, Enseñanza, Evaluación. Madrid. Instituto Cervantes. 2002-2004.

DE SALVADOR, Nuria. Developing Students' Multiple Intelligences. New York: Scholastic Professional Books 1998. 